\title{
Long-term habituation of the gill-withdrawal reflex in Aplysia requires gene transcription, calcineurin and L-type voltage-gated calcium channels
}

\author{
Joseph Esdin ${ }^{1+}$, Kaycey Pearce ${ }^{1}$ and David L. Glanzman ${ }^{1,2,3 *}$ \\ 1 Department of Integrative Biology and Physiology, University of California, Los Angeles, CA, USA \\ 2 Department of Neurobiology and the Brain Research Institute, David Geffen School of Medicine, University of California, Los Angeles, CA, USA \\ ${ }^{3}$ Marine Biological Laboratory, Woods Hole, MA, USA
}

\section{Edited by:}

Jean-Christophe Sandoz, Centre National De La Recherche Scientifique, France

\section{Reviewed by:}

Thomas Abrams, University of Maryland School of Medicine, USA György Kemenes, University of Sussex, UK

\section{*Correspondence:}

David L. Glanzman, Gonda (Goldschmied) Neuroscience and Genetics Research Center, University of California, 695 Charles E. Young

Drive South, Box 951761, Los Angeles, CA 90095-1761, USA.

e-mail:dglanzman@physci.ucla.edu

${ }^{+}$Present address:

Joseph Esdin, Math, Science and Engineering Division, Antelope Valley College, Lancaster, CA, USA
Although habituation is possibly the simplest form of learning, we still do not fully understand the neurobiological basis of habituation in any organism. To advance the goal of a comprehensive understanding of habituation, we have studied long-term habituation (LTH) of the gill-withdrawal reflex (GWR) in the marine snail Aplysia californica. Previously, we showed that habituation of the GWR in a reduced preparation lasts for up to $12 \mathrm{~h}$, and depends on protein synthesis, as well as activation of protein phosphatases 1 and $2 \mathrm{~A}$ and postsynaptic glutamate receptors. Here, we have used the reduced preparation to further analyze the mechanisms of LTH in Aplysia. We found that LTH of the GWR depends on RNA synthesis because it was blocked by both the irreversible transcriptional inhibitor actinomycin-D and the reversible transcriptional inhibitor, 5,6dichlorobenzimidazole riboside (DRB). In addition, LTH requires activation of protein phosphatase 2B (calcineurin), because it was disrupted by ascomycin. Finally, LTH was blocked by nitrendipine, which indicates that activation of L-type voltage-gated $\mathrm{Ca}^{2+}$ channels is required for this form of learning. Together with our previous results, the present results indicate that exclusively presynaptic mechanisms, although possibly sufficient for short-term habituation, are insufficient for LTH. Rather, LTH must involve postsynaptic, as well as presynaptic, mechanisms.

Keywords: behavior, non-associative learning, long-term memory, habituation, long-term depression, mollusk

\section{INTRODUCTION}

Habituation is a response decrement to the repeated application of a given stimulus that cannot be attributed to sensory adaptation, or sensory or motor fatigue (Thompson and Spencer, 1966; Rankin et al., 2009). Although apparently ubiquitous throughout the animal kingdom, and commonly regarded as the simplest form of learning, habituation remains poorly understood with respect to its underlying neuronal mechanisms, particularly in vertebrates. Some progress has been made, however, toward an understanding of the neural basis of habituation in simpler invertebrate organisms, particularly the crayfish (Krasne and Teshiba, 1995), the mollusk Aplysia (Glanzman, 2009), and the nematode Caenorhabditis elegans (Giles and Rankin, 2009). Nonetheless, even in these relatively simple organisms, there are large gaps in our understanding of the cell biology of habituation.

Habituation can exhibit both short- and long-term forms. In Aplysia the siphon-elicited gill- and siphon-withdrawal reflex can undergo habituation that persists for several weeks (Carew et al., 1972). This form of long-term habituation (LTH) is characterized by long-term synaptic depression of the sensorimotor pathway that mediates the withdrawal reflex (Carew and Kandel, 1973; Castellucci et al., 1978), as well as by retraction of the presynaptic terminals and branches of siphon sensory neurons (Bailey and Chen, 1983, 1988a). However, the signaling pathways whose activation during LTH triggers these long-term cellular changes remain fairly obscure. In a previous study (Ezzeddine and Glanzman, 2003) we used a reduced preparation to study the signaling pathways involved in LTH of the gill-withdrawal reflex (GWR) in Aplysia. We found that LTH of the GWR depends on protein synthesis, as well as activation of protein phosphatases 1 and $2 \mathrm{~A}$ and postsynaptic glutamate receptors, specifically, $\alpha$-amino-3-hydroxy-5-methyl4-isoxazolepropionic acid (AMPA) and $N$-methyl-D-aspartate (NMDA) receptors. Here, we used our reduced preparation to test the roles of RNA synthesis, calcineurin activity and L-type $\mathrm{Ca}^{2+}$ channel activation in LTH in Aplysia.

\section{MATERIALS AND METHODS}

We have previously described the Materials and Methods in detail (Ezzeddine and Glanzman, 2003). Briefly, adult Aplysia californica (75-150 g) were obtained from a local supplier (Alacrity Marine, Redondo Beach, CA, USA) and housed for $\geq 24 \mathrm{~h}$ prior to the start of the experiments. During an experiment the animal was initially anesthetized with isotonic $\mathrm{MgCl}_{2}$, and then the mantle shelf, gill, siphon, and tail were dissected away from the rest of the body, together with the CNS (minus the buccal ganglia), which was left connected to the siphon and gill via the siphon and branchial nerves, respectively. The abdominal artery was cannulated with polyethylene tubing ( 0.024 in OD, 0.011 in ID, Intramedic, Parsippany, NJ, USA), and this was connected to a peristaltic pump. During experiments the abdominal artery was perfused $($ rate $=1.5 \mathrm{ml} / \mathrm{hr})$ with aerated normal artificial seawater $\left(\mathrm{ASW}, 15^{\circ} \mathrm{C}\right.$ ) via the cannula. The cannula was also used to selectively administer drugs to 
the abdominal ganglion. Following cannulation of the abdominal artery, the preparation was pinned to the Sylgard-lined bottom of a Lucite experimental chamber. The siphon was left unpinned. The afferent vein of the gill was cannulated with polyethylene tubing and perfused with chilled, aerated ASW. The cannula in the afferent vein was secured with a surgical silk suture, and the suture was connected to a force transducer (Model 1040 or 1030, ADInstruments, Grand Junction, CO, USA), which was used to measure the GWR. Habituating and test stimuli were delivered to the siphon via pairs of Teflon-insulated platinum wires $(0.005 \mathrm{~mm}$ in diameter, \#773000, AM Systems, Carlsborg, WA, USA). One wire was inserted into each side of the siphon, and a ground wire was placed in the bath. The intensity of the stimuli $(500 \mathrm{~ms}$ trains [ $25 \mathrm{~Hz}$ ] of $10 \mathrm{~ms}$ current pulses) was set for each preparation to be just suprathreshold for reliably eliciting gill withdrawal, and remained fixed throughout an experiment. One side of the siphon (Trained) received both the test and habituating stimulation, whereas the other side (Untrained) received only the test stimulation.

After the intensity of the stimuli had been determined for the Trained and Untrained sides, the preparation was rested for 60-90 $\mathrm{min}$. At the end of the rest period a single stimulus was delivered to the Trained side of the siphon. Preparations that did not respond to this initial stimulus with a visible gill contraction were discarded. Furthermore, data from a preparation were accepted only if the posttest response of the Untrained side was at least 80\% of the pretest response.

All drugs were purchased from Sigma Aldrich (St. Louis, MO, USA). The drugs were initially dissolved in DMSO, and then diluted to their final concentrations in artificial seawater (ASW). (The final concentration of DMSO in the infusate was $0.1-0.2 \%$.) The drugs were directly delivered to the abdominal ganglion via the cannula in the abdominal artery. The irreversible transcriptional inhibitor actinomycin-D was infused into the abdominal ganglion for $1 \mathrm{~h}$ prior to the pretests, and then washed out. All other drugs were infused into the abdominal ganglion starting immediately after the pretests, and then washed out of the abdominal ganglion with ASW prior to the posttests. In control preparations ASW, containing the same concentration of DMSO as the infusate used in the matched experimental preparations, was infused into the abdominal artery, and then washed out with normal ASW prior to the posttest. The experimental preparations and the control preparations used to test the effect of an experimental drug on habituation were from animals that were all collected at the same time.

The side of the siphon that was chosen to be the Trained side was alternated systematically between left and right sides. The Trained side initially received a pretest stimulus, and $5 \mathrm{~min}$ later a pretest stimulus was delivered to the other side of the siphon (Untrained side). $1 \mathrm{~h}$ after the pretest stimulus to the Untrained side, the Trained side received habituation training, which consisted of five blocks of stimuli (interblock interval $=90 \mathrm{~min}$ ). During each block 30 stimuli (ISI $=30$ or $60 \mathrm{~s}$ ) were delivered to the Trained side. Following a $2 \mathrm{~h}$ rest period after habituation training, the Trained and Untrained sides each received a single posttest stimulus. The two posttest stimuli were separated by $5 \mathrm{~min}$.

The peak amplitude (maximum-minimum) of each gill contraction was determined using a digital data acquisition system (MacLab 4 s/2e or PowerLab 8 s, ADInstruments, Colorado Springs,
CO, USA). The minimum was the base line response recorded when the gill was in a relaxed state $1 \mathrm{~s}$ prior to stimulation. The pretest value of the GWR was set to be equal to $100 \%$ for the Trained and Untrained sides, and subsequent GWRs were normalized to the pretest value. All responses in the Results are expressed as the mean normalized response \pm SEM. Non-parametric statistical tests were used for all comparisons. Wilcoxon tests were used for paired comparisons, and Mann-Whitney tests were used for unpaired comparisons unless otherwise indicated. All significance values reported represent two-tailed levels of significance.

\section{RESULTS \\ LONG-TERM HABITUATION DEPENDS ON RNA SYNTHESIS}

To test whether LTH of the GWR requires transcription, actinomycin-D (40 $\mu \mathrm{M}$ in ASW with $0.2 \%$ DMSO) was infused into the abdominal ganglion. Actinomycin-D's effects are irreversible; therefore, the drug was applied for $1 \mathrm{~h}$, and then washed out with ASW immediately before the start of the pretests. The actinomycin-D treatment $(n=6)$ blocked LTH (Figure 1A). The difference between the pretest and posttest withdrawal in response to stimulation of the Trained side was not significant (mean normalized posttest response $=99.3 \pm 12.5 \%, p>0.6$ ). Furthermore, the posttest GWR evoked by stimulation of the Untrained siphon side was not significantly different from the pretest GWR (mean normalized posttest response $=111.2 \pm 9.5 \%, p>0.1$ ). Finally, there was no statistical difference between the Trained and Untrained posttest GWRs $(p>0.1)$.

In the control experiments $(n=6)$ ASW containing $0.2 \%$ DMSO was infused into the abdominal ganglion $1 \mathrm{~h}$ before the pretests, and washed out immediately before the start of the experiment. The training produced LTH of the GWR to Trained side stimulation (mean normalized posttest response $=47.3 \pm 10.2 \%, p<0.04$ for the comparison with the Trained pretest response) (Figure 1B). By contrast, the posttest GWR to stimulation of the Untrained side of the siphon did not differ significantly from the pretest GWR (mean normalized response $=108 \pm 8.8 \%, p>0.4$ ). Also, the posttest response evoked by Trained side stimulation was significantly less than that evoked by Untrained side stimulation $(p<0.003)$. In addition, there was a significant difference between the Trained posttest GWRs of the actinomycin-D-treated and control groups $(p<0.003)$. The Untrained posttest responses of the two groups, however, did not differ significantly $(p>0.8)$. Importantly, despite the fact that actinomycin-D treatment blocked LTH, Trained-site stimulation nonetheless produced normal short-term habituation $(\mathrm{STH})$ of the GWR in the actinomycin-treated preparations. (Compare the Trained responses to the Block 1 habituating stimuli in Figures 1A,B).

To control for non-specific effects of actinomycin-D, we tested the effect of a reversible transcriptional inhibitor, 5,6-dichloro-1$\beta$-D-ribofuranosylbenzimidazole (DRB) (Sehgal and Tamm, 1978; Yamaguchi et al., 1998; Apergis-Schoute et al., 2005), on LTH. DRB ( $150 \mu \mathrm{M}$ in ASW with $0.2 \%$ DMSO) was present in the abdominal ganglion throughout the training period. The presence of DRB during habituation training blocked LTH ( $n=9$, Figure 2A). There was no significant change in the Trained GWR (mean normalized postest response $=115.1 \pm 10.9 \%, p>0.2$ for the comparison with the pretest response). The GWR to stimulation of the Untrained 

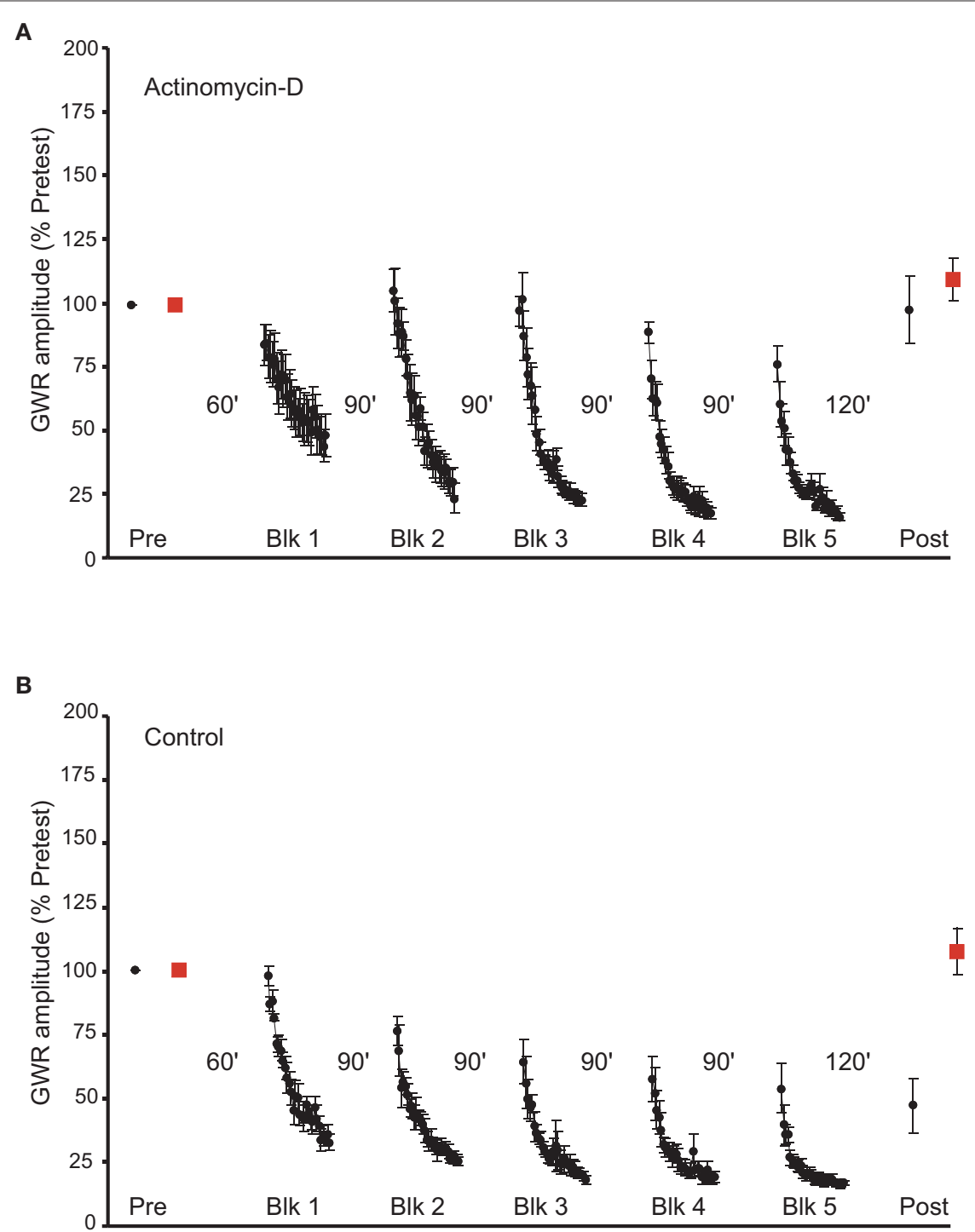

FIGURE 1 | Effect of actinomycin-D treatment on LTH. (A) Experiments in which preparations were treated with the irreversible transcriptional inhibitor actinomycin-D prior to habituation training $(n=6)$. The drug blocked the induction of LTH. (B) Data for control experiments $(n=6)$. Here, the abdominal ganglion was perfused with normal ASW plus DMSO for the time corresponding to the period of actinomycin-D treatment. The habituation training produced significant LTH of the GWR to stimulation of the Trained side of the siphon. In this and the following figures the solid circles represent the responses to Trained side stimulation, whereas the solid squares represents the responses to Untrained side stimulation. side also did not change (mean normalized Untrained posttest response $=122.6 \pm 19.1 \%, p>0.7$ for the comparison with the pretest response). In addition, the Trained and Untrained posttests did not differ $(p>0.8)$. Control experiments, in which ASW with $0.2 \%$ DMSO was infused into the abdominal artery, were performed using the same protocol as the DRB experiments. The habituation training produced significant LTH of the Trained GWR in the control experiments $(n=9$, Figure $2 \mathbf{B})$. There was a significant decrease in posttest response to Trained side stimulation (mean normalized posttest response $=75.6 \pm 8.8 \% p<0.04$ for the comparison with the pretest response). However, there was a significant increase in the GWR to stimulation of the Untrained side (mean normalized posttest response $=118.4 \pm 7.5 \%, p<0.03$ for the comparison with the Untrained pretest response). Furthermore, the posttest Untrained GWR was significantly greater than the posttest Trained GWR $(p<0.004)$. Finally, the posttest GWRs to Trained side stimulation differed significantly in the DRB-treated and control preparations $(p<0.02)$.

The effect of the habituation training on the GWR to Untrained side stimulation in the control experiments suggests that the training produced some generalized sensitization, as well as pathwayspecific LTH, in these experiments. In our earlier study (Ezzeddine and Glanzman, 2003) we also observed that the GWR to Untrained side stimulation sometimes exhibited sensitization after habituation training, and that there was often sensitization of the GWR to Trained side stimulation during training in the presence of drugs 

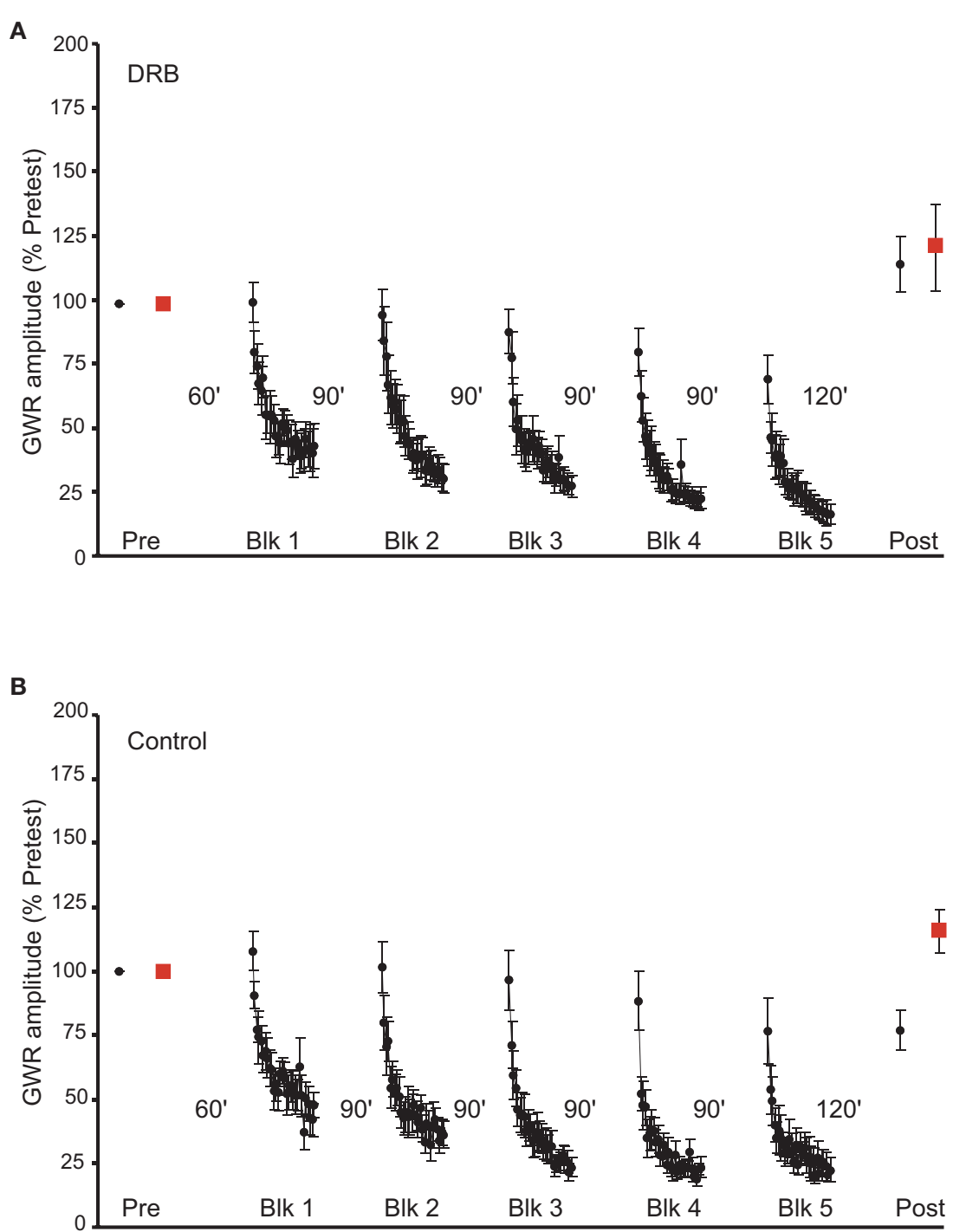

FIGURE 2 | Effect of DRB treatment on LTH. (A) Data from experiments ( $n=9)$ in which preparations were treated with the reversible transcriptional inhibitor DRB during habituation training. DRB infusion disrupted LTH. (B) Data for the control experiments $(n=9)$. Here, training was carried out in the presence of ASW plus DMSO, which produced LTH on the Trained side and sensitization on the Untrained side.

that blocked LTH. We believe, as discussed in that paper, that these phenomena reflect the fact that the habituating stimuli can activate both habituating and sensitizing neuronal processes, as originally proposed by Groves and Thompson (1970). According to Groves and Thompson, the ultimate behavioral outcome, whether habituation, sensitization, or no change, is determined by the relative strengths of these two countervailing processes. It should be noted, however, that both the sensitization of the GWR on the Untrained side, and the sensitization of the reflex on the Trained side during habituation training in the presence of inhibitors of LTH (e.g., Blocks 2 and 3, Figure 1A), were highly variable and not always observed.

\section{LONG-TERM HABITUATION REQUIRES CALCINEURIN ACTIVITY}

We previously found that LTH depends on activation of NMDA-type receptors in abdominal ganglion. Activation of postsynaptic NMDA receptors would be expected to produce a rise in intracellular $\mathrm{Ca}^{2+}$ in neurons of the GWR circuit. The $\mathrm{Ca}^{2+} /$ calmodulin-dependent phosphatase calcineurin has been shown to be involved in both long-term synaptic depression (Mulkey et al., 1994) and memory extinction (Baumgartel et al., 2008) in mammals. Given that long-term depression (LTD) of the sensorimotor synapse (Lin and Glanzman, 1996) is a candidate mechanism for LTH in Aplysia (see Ezzeddine and Glanzman, 2003; Glanzman, 2009), we wished to know whether LTH was regulated by calcineurin activity. Accordingly, we tested the effect of ascomycin $(10 \mu \mathrm{M}$ in ASW with $0.2 \%$ DMSO), a selective calcineurin inhibitor (Sierra-Paredes and Sierra-Marcuño, 2008), on LTH. Ascomycin application during habituation training blocked LTH ( $n=7$, Figure 3A). The difference between the pretest and posttest responses for Trained side stimulation was not significant (mean normalized posttest response $=104.2 \pm 14 \%, p>0.9$ ). However, as was the case for the control experiments in Figure $2 \mathbf{B}$, the posttest response for 

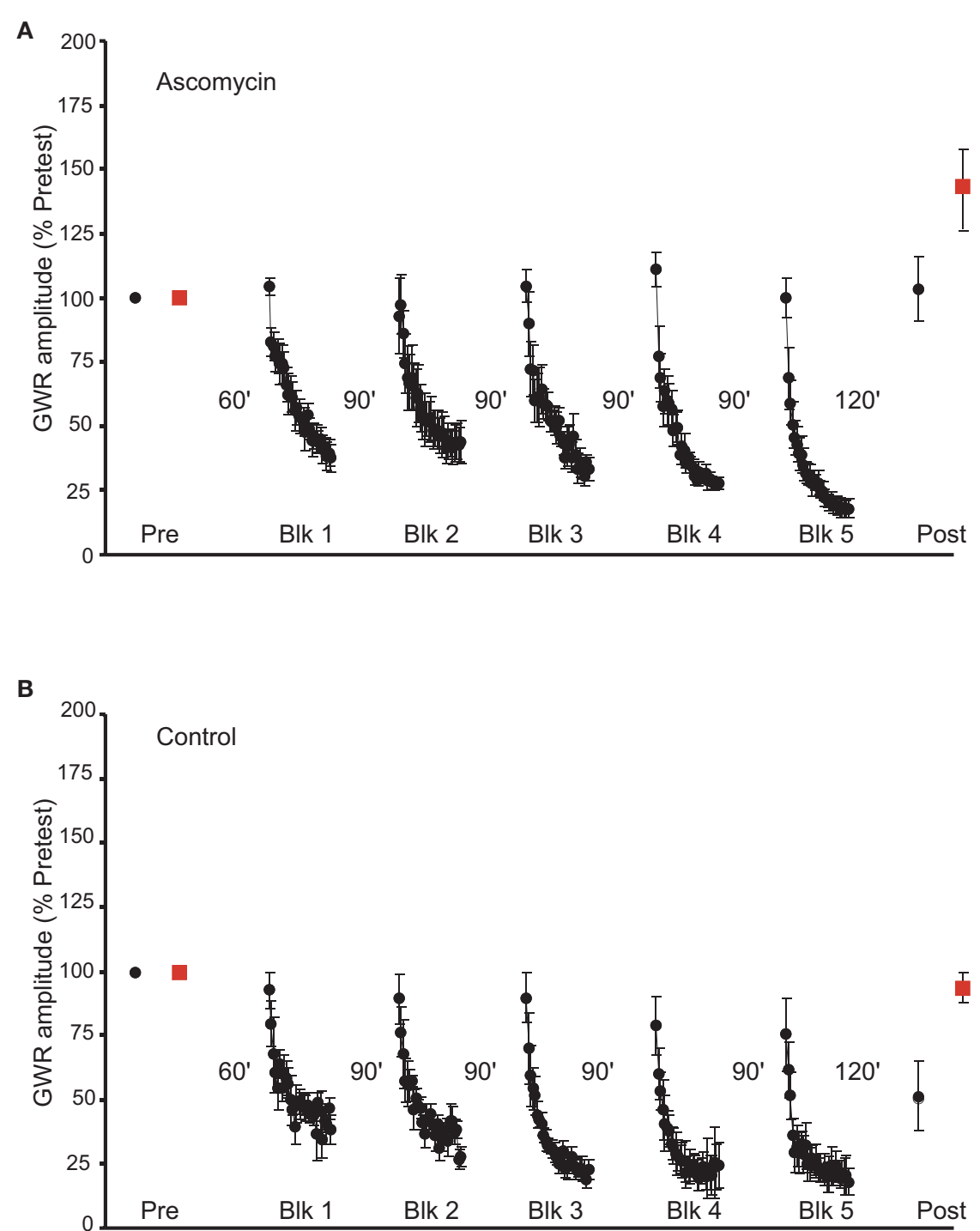

FIGURE 3 | Effect of inhibition of calcineurin on LTH. (A) Data from experiments $(n=7)$ in which the abdominal ganglion was infused with ascomycin during habituation training. Inhibition of calcineurin blocked LTH. (B) Control data from experiments $(n=5)$ in which a control drug, rapamycin, which does not inhibit protein phosphatases, was present in the abdominal ganglion during training. Training in the presence of rapamycin produced LTH of the GWR to Trained side stimulation.

the Untrained side exhibited significant enhancement (mean normalized posttest response $=143.2 \pm 15.7 \%, p<0.05$ for the comparison with the pretest response). The difference between the posttest responses for the Trained and the Untrained sides, however, was not significant $(p>0.1)$. Control experiments $(n=5)$ were performed with rapamycin (10 $\mu \mathrm{M}$ in ASW with $0.2 \%$ DMSO) present in the cannula during training (Figure 3B). Rapamycin has the same binding site on calcineurin as ascomycin, but rapamycin does not inhibit dephosphorylation by calcineurin (Schreiber and Crabtree, 1992). In contrast to the effect of ascomycin, rapamycin did not affect LTH. Thus, habituation training resulted in a significantly reduced GWR for the Trained side (mean normalized posttest response $=51.4 \pm 13 \%, p<0.03$ [ $t$-test used here for the comparison with the pretest response]). The difference between the pretest and posttests responses for Untrained side, however, was not significant (mean normalized posttest GWR $=93.4 \pm 6.7 \%, p>0.3$ ). As would be expected from these results, the posttest responses for the Trained and Untrained sides differed significantly in the experiments involving rapamycin treatment $(p<0.03)$. Finally, the posttest GWR to Trained side stimulation was significantly less in the rapamycin-treated preparations than in the ascomycintreated preparations $(p<0.05)$. Notice that, although blockade of calcineurin activity with ascomycin disrupted LTH, it did not affect STH (Figure 3A).

\section{ACTIVATION OF L-TYPE VOLTAGE-GATED $\mathrm{CA}^{2+}$ CHANNELS APPEARS TO BE NECESSARY FOR LTH}

We previously found that activation of AMPA receptors, as well as of NMDA receptors, contributes to LTH of the GWR (Ezzeddine and Glanzman, 2003). It is therefore possible that 
depolarization-induced activation of L-type voltage-gated $\mathrm{Ca}^{2+}$ channels (LVGCCs) is upstream of calcineurin activity during LTH. To test this possibility, we examined the effect of blockade of LVGCCs on LTH. We infused nitrendipine $(100 \mu \mathrm{M}$ in ASW with $0.14 \%$ DMSO), an antagonist of LVGCCs (Bolshakov and Siegelbaum, 1994), into the abdominal ganglion during habituation training $(n=6)$. Nitrendipine treatment blocked LTH to stimulation of the Trained side of the siphon (mean normalized posttest response $=116.8 \pm 13 \%, p>0.3$ for the comparison with the pretest response) (Figure 4A). In these experiments habituation training did not alter the GWR to stimulation of the Untrained side; the mean normalized Untrained posttest response ( $157.8 \pm 39.4 \%$, was not significantly different from the mean normalized Untrained pretest response $(p>0.4)$, although the variability in the Untrained responses was admittedly large. Furthermore, the GWR evoked by posttest stimulation of the Trained side was not significantly different from that evoked by posttest stimulation of the Untrained side $(p>0.9)$. In control experiments $(n=8)$, performed at the same time as the nitrendipine experiments, the abdominal ganglion was infused ASW plus $0.14 \%$ DMSO. Here, habituation training produced significant LTH of the GWR to stimulation of the Trained side (mean normalized posttest response $=55.1 \pm 12.4 \%, p<0.02$ for the comparison with the pretest response) (Figure 4B). There was no significant change in the GWR to stimulation of the Untrained side (mean Untrained normalized posttest response $=124.4 \pm 15.3 \%$, $p>0.1$ for the comparison with the Untrained pretest response). Furthermore, the Trained side posttest GWR and the Untrained side posttest GWR differed significantly $(p<0.004)$. Finally, the difference between the posttest response to the Trained side stimulation in the nitrendipine group and that in the control group was highly significant $(p<0.005)$. These results support the idea that LVGCC activity is essential for LTH, although such activity appears not to be necessary for STH (Figure 4A).

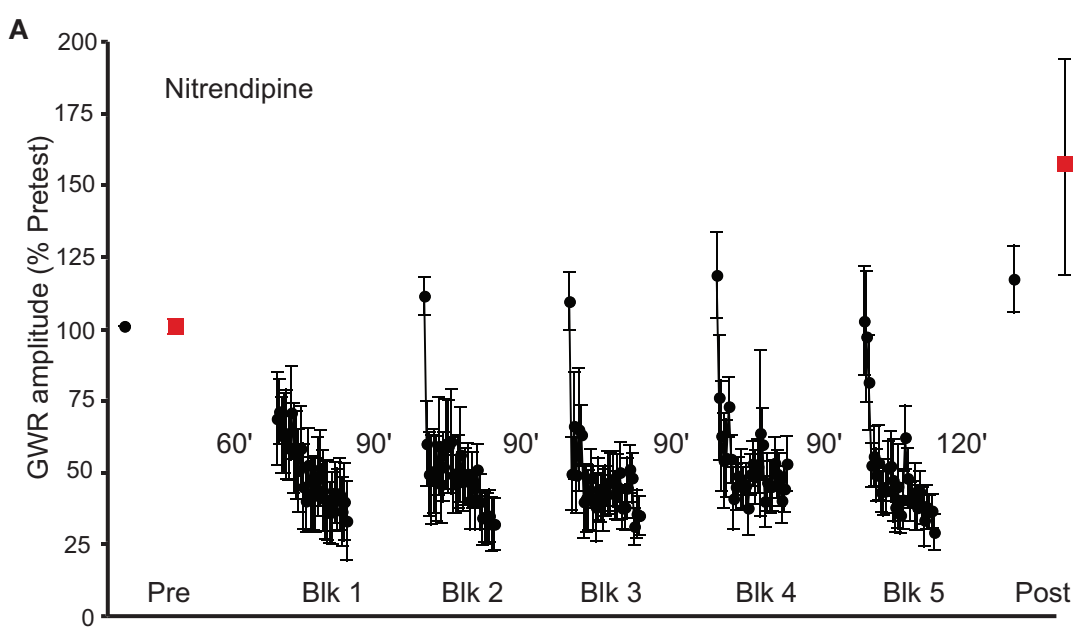

B

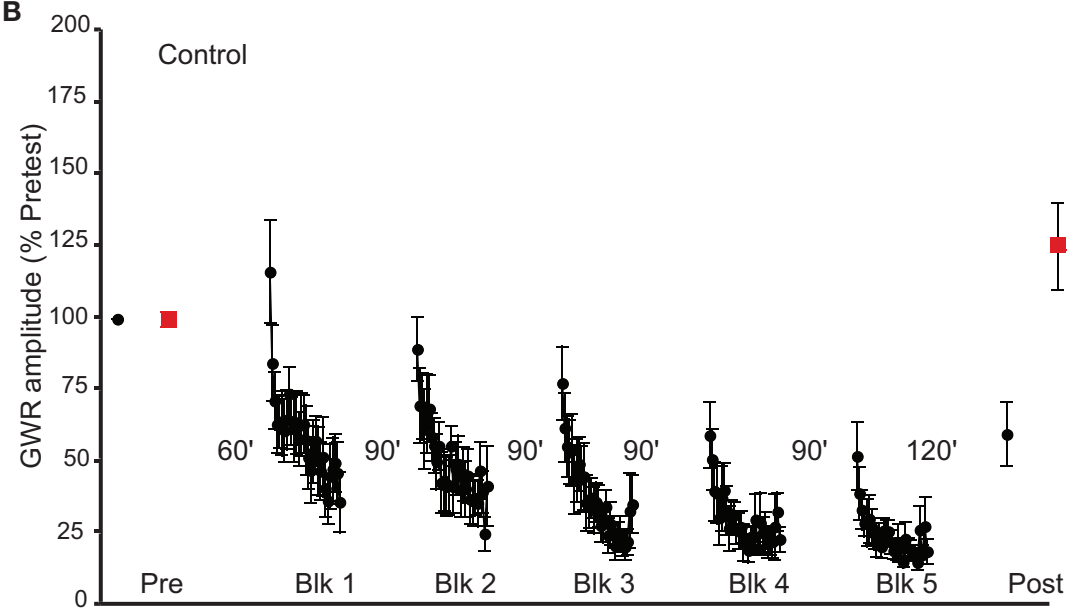

FIGURE 4 | Effect of inhibiting LVGCCs on LTH. (A) Data from experiments $(n=6)$ in which nitrendipine was present during habituation training. The drug blocked the induction of LTH. (B) Data from control experiments $(n=8)$ in which training occurred in the presence of ASW plus DMSO. The training resulted in LTH of the GWR. 


\section{DISCUSSION \\ ROLE OF GENE TRANSCRIPTION IN LTH IN APLYSIA}

Memory in Aplysia has been divided into three stages: short-term, intermediate-term and long-term (Goelet et al., 1986; Sutton and Carew, 2002). These three stages are distinguished by both their temporal and mechanistic properties. Temporally - although their time courses overlap somewhat - short-term memory (STM) lasts from seconds to $\sim 30 \mathrm{~min}$; intermediate-term memory (ITM) lasts from $\sim 30 \mathrm{~min}$ to $3 \mathrm{~h}$; and long-term memory lasts for $>10 \mathrm{~h}$. Mechanistically, STM depends exclusively on posttranslational changes; ITM requires protein synthesis, but not gene transcription; and LTM requires both translation and transcription. However, most of the previous work characterizing the mechanisms underlying the different phases of memory in Aplysia has concerned only one type of memory - sensitization of the withdrawal reflex. Although an early report demonstrated that a neural correlate of STH of the GWR did not require protein synthesis (Schwartz et al., 1971), there has been little attempt until recently to determine whether there are different phases of memory for habituation that correspond mechanistically to those for sensitization. We previously showed that the habituation of the GWR that persisted for 1-6 h following a $5 \mathrm{~h}$ period of spaced training required protein synthesis (Ezzeddine and Glanzman, 2003). But in that study we did not test whether this form of habituation memory also required gene transcription. Here, we have shown that the habituation resulting from a similar training protocol requires transcription, because it was blocked by actinomycin-D and DRB. Therefore, this form of habituation fits the definition of LTM as it has commonly been defined in Aplysia (Goelet et al., 1986). It will be interesting in the future to determine whether there is a training protocol that produces an intermediate form of habituation memory, one that requires translation, but not transcription.

\section{HOMOSYNAPTIC DEPRESSION CANNOT ACCOUNT FOR LTH IN APLYSIA}

It has long been believed that the mechanism of habituation of the gill- and siphon-withdrawal reflex is due exclusively to presynaptic changes. This mechanism, homosynaptic depression (HSD), is thought to involve decreased transmitter release from presynaptic terminals due to repeated activation of sensory neurons (Castellucci and Kandel, 1974; Armitage and Siegelbaum, 1998). Currently, the mechanism underlying HSD in Aplysia is controversial. It was originally proposed that HSD was caused by depletion of the readily releasable pool of presynaptic vesicles (Gingrich and Byrne, 1985; Hochner et al., 1986; Bailey and Chen, 1988b). More recent evidence, however, indicates that HSD results from all-or-none silencing of presynaptic release sites (Gover and Abrams, 2009).

Although HSD may indeed be a major mechanism of STH, the data from this and our previous study (Ezzeddine and Glanzman, 2003) argue strongly against the notion that LTH can be explained by exclusively presynaptic changes. First, HSD does not depend on glutamate receptor activity (Armitage and Siegelbaum, 1998), whereas LTH does (Ezzeddine and Glanzman, 2003). Second, LTH requires activation of LVGCCs (present study), which is most likely caused by AMPA receptor-mediated postsynaptic depolarization (see Ezzeddine and Glanzman, 2003). Studies by Bailey and Chen (1983, 1988a) indicate that LTH is accompanied by presynaptic morphological changes, including fewer vesicles in sensory neuron-associated active zones, and a decrease in the number of varicosities per sensory neuron. These morphological data, together with our data, support the conclusion that LTH involves both preand postsynaptic long-term cellular changes. If correct, this conclusion raises the intriguing possibility that the pre- and postsynaptic changes are coordinated by transsynaptic signals (see Glanzman, 2009, for further discussion).

\section{ROLE OF CALCINEURIN AND LVGCCS IN LTH}

The data from the present study suggest that increased intracellular $\mathrm{Ca}^{2+}$ plays a key role in LTH. One likely source of elevated intracellular $\mathrm{Ca}^{2+}$ is influx through postsynaptic NMDA receptors (Ezzeddine and Glanzman, 2003). Activated LVGCCs, due to AMPA receptor-mediated postsynaptic depolarization, represent another potential source of the elevated intracellular $\mathrm{Ca}^{2+}$ necessary for LTH. Our finding that ascomycin blocks the induction of LTH indicates that calcineurin is one of the proteins whose activity is stimulated by increased intracellular $\mathrm{Ca}^{2+}$. Interestingly, calcineurin activity has been implicated in both LTD (Mulkey et al., 1994) and depotentiation (Jouvenceau et al., 2003) in the mammalian brain, as well as in some forms of extinction in mammals (Lin et al., 2003; Baumgartel et al., 2008). We do not yet know what downstream pathways are stimulated by calcineurin during LTH. One action linked with calcineurin activity in the mammalian brain is endocytosis of AMPA receptors, which is believed to be a key event in LTD (Beattie et al., 2000). Endocytosis of AMPA-type receptors could well be one effect of calcineurin activity during habituation of the GWR (see Glanzman, 2009), because exocytosis of AMPAtype receptors has previously been shown to play a role in synaptic facilitation and enhancement of the withdrawal reflex in Aplysia (Chitwood et al., 2001; Li et al., 2005, 2009).

\section{POTENTIAL ROLE OF LTD IN LTH IN APLYSIA}

The synaptic mechanisms that underlie LTH of the withdrawal reflex remain to be identified. One potential mechanism that is consistent with the data in this and our previous study (Ezzeddine and Glanzman, 2003) is LTD induced by elevated postsynaptic $\mathrm{Ca}^{2+}$ (Malenka and Bear, 2004). A form of LTD of the sensorimotor synapse that depends partly on elevated postsynaptic $\mathrm{Ca}^{2+}$ has been demonstrated in dissociated cell culture (Lin and Glanzman, 1996); but this form of synaptic depression has not yet been experimentally linked to LTH. A problem for the idea that activity-dependent LTD is a mechanism of LTH is that induction of activity-dependent LTD requires $1 \mathrm{~Hz}$ electrical stimulation, which is quite different from the rate of behavioral stimulation that results in LTH. Further complicating the issue of the synaptic mechanism of LTH is the demonstration by Montarolo et al. (1988) that homosynaptic activation of the sensorimotor synapse with a rate of stimulation designed to mimic the synaptic stimulation that occurs during LTH training fails to induce long-term $(24 \mathrm{~h})$ depression of the synapse. The apparent failure of naturalistic homosynaptic activity to induce LTD of the sensorimotor synapse suggests that homosynaptic activation of the sensorimotor pathway, by itself, may be insufficient for LTH in Aplysia. The possibility that LTH may depend, at least in part, on one or more heterosynaptic pathways is supported by the finding that spaced applications of the endogenous inhibitory neuropeptide, FMRFamide, can produce depression of the 
sensorimotor synapse that persists for $\geq 24 \mathrm{~h}$ (Montarolo et al., 1988). Both heterosynaptic, inhibitory (GABAergic), and homosynaptic pathways have been shown to play roles in habituation of the crayfish tailflip escape response (Krasne and Teshiba, 1995).

\section{MECHANISTIC SIMILARITIES BETWEEN HABITUATION AND EXTINCTION}

Habituation, like extinction, is the waning of a response due to repeated presentation of an unchanging stimulus. The major phenomenological distinction between habituation and extinction is that habituation is the waning of an innate response, whereas extinction is the waning of a conditioned response. Despite this distinction, the behavioral similarity between habituation and extinction has led to the speculation that these two forms of memory may share common mechanisms (McSweeney and Swindell, 2002). Although controversial, there is experimental

\section{REFERENCES}

Apergis-Schoute, A. M., Debiec, J., Doyere, V., LeDoux, J. E., and Schafe, G. E. (2005). Auditory fear conditioning and long-term potentiation in the lateral amygdala require ERK/MAP kinase signaling in the auditory thalamus: a role for presynaptic plasticity in the fear system. J. Neurosci. 25 , 5730-5739.

Armitage, B. A., and Siegelbaum, S. A. (1998). Presynaptic induction and expression of homosynaptic depression at Aplysia sensorimotor neuron synapses. J. Neurosci. 18, 8770-8779.

Bailey, C. H., and Chen, M. (1983). Morphological basis of long-term habituation and sensitization in Aplysia. Science 220, 91-93.

Bailey, C. H., and Chen, M. (1988a). Longterm memory in Aplysia modulates the total number of varicosities of single identified sensory neurons. Proc. Natl. Acad. Sci. U.S.A. 85, 2373-2377.

Bailey, C. H., and Chen, M. (1988b). Morphological basis of short-term habituation in Aplysia. J. Neurosci. 8, 2452-2459.

Barad, M., Blouin, A. M., and Cain, C. K. (2004). Like extinction, latent inhibition of conditioned fear in mice is blocked by systemic inhibition of L-type voltage-gated calcium channels. Learn. Mem. 11, 536-539.

Baumgartel, K., Genoux, D., Welzl, H., Tweedie-Cullen, R. Y., Koshibu, K., Livingstone-Zatchej, M., Mamie, C., and Mansuy, I. M. (2008). Control of the establishment of aversive memory by calcineurin and Zif268. Nat. Neurosci. 11, 572-578.

Beattie,E.C.,Carroll,R.C.,Yu,X., Morishita, W., Yasuda, H., von Zastrow, M., and Malenka, R. C. (2000). Regulation of AMPA receptor endocytosis by a signaling mechanism shared with LTD. Nat. Neurosci. 3, 1291-1300.

Bolshakov, V. Y., and Siegelbaum, S. A. (1994). Postsynaptic induction and presynaptic expression of hippocampal long-term depression. Science 264 , 1148-1152.

Cain, C. K., Godsil, B. P., Jami, S., and Barad, M. (2005). The L-type calcium channel blocker nifedipine impairs extinction, but not reduced contingency effects, in mice. Learn. Mem. $12,277-284$.

Carew, T. J., and Kandel, E. R. (1973) Acquisition and retention of longterm habituation in Aplysia: correlation of behavioral and cellular processes. Science 182, 1158-1160.

Carew, T.J., Pinsker, H. M., and Kandel, E. R. (1972). Long-term habituation of a defensive withdrawal reflex in Aplysia. Science 175, 451-454.

Castellucci, V. F., Carew, T. J., and Kandel, E. R. (1978). Cellular analysis of longterm habituation of the gill-withdrawal reflex of Aplysia californica. Science 202, 1306-1308.

Castellucci, V. F., and Kandel, E. R. (1974). A quantal analysis of the synaptic depression underlying habituation of the gill-withdrawal reflex in Aplysia. Proc. Natl.Acad. Sci.U.S.A.71, 5004-5008.

Chitwood, R. A., Li, Q., and Glanzman, D L. (2001). Serotonin facilitates AMPAtype responses in isolated siphon motor neurons of Aplysia in culture. J. Physiol. 534, 501-510.

Ezzeddine, Y., and Glanzman, D. L. (2003). Prolonged habituation of the gill-withdrawal reflex in Aplysia depends on protein synthesis, protein phosphatase activity, and postsynaptic glutamate receptors. J. Neurosci. 23 , 9585-9594.

Giles, A. C., and Rankin, C. H. (2009) Behavioral and genetic characterization of habituation using Caenorhabditis elegans. Neurobiol. Learn. Mem. 92, 139-146.

Gingrich, K. J., and Byrne, J. H. (1985). Simulation of synaptic depression, posttetanic potentiation, and presy-

support for this idea (Kamprath et al., 2006). We have found that LTH in Aplysia requires the activity of calcineurin and LVGCCs. Both of these processes have also been implicated in extinction (Lin et al., 2003; Barad et al., 2004; Suzuki et al., 2004, 2008; Cain et al., 2005; Baumgartel et al., 2008) (but see Schafe, 2008). Possibly, therefore, an understanding of the cellular mechanisms that mediate LTH of the GWR in Aplysia will contribute important mechanistic insights into the considerably more complex phenomenon of extinction.

\section{ACKNOWLEDGMENTS}

We thank A. Roberts and D. Cai for helpful comments on the manuscript. This work was supported by grants R01 MH068543 and R37 NS029563 from the US National Institutes of Health, and a grant from the Dart Foundation (David L. Glanzman).

naptic facilitation of synaptic potentials from sensory neurons mediating gill-withdrawal reflex in Aplysia. $J$ Neurophysiol. 53, 652-669.

Glanzman, D. L. (2009). Habituation in Aplysia: the cheshire cat of neurobiology. Neurobiol. Learn. Mem. 92 147-154.

Goelet,P., Castellucci,V.F.,Schacher,S., and Kandel, E. R. (1986). The long and the short of long-term memory-a molecular framework. Nature 322, 419-422.

Gover, T. D., and Abrams, T. W. (2009) Insights into a molecular switch that gates sensory neuron synapses during habituation in Aplysia. Neurobiol. Learn. Mem. 92, 155-165.

Groves, P.M., and Thompson, R. F. (1970) Habituation: a dual-process theory. Psychol. Rev. 77, 419-450.

Hochner, B., Klein, M., Schacher, S., and Kandel, E. R. (1986). Additional component in the cellular mechanism of presynaptic facilitation contributes to behavioral dishabituation in Aplysia. Proc. Natl. Acad. Sci. U.S.A 83, 8794-8798.

Jouvenceau, A., Billard, J.-M., Haditsch, U., Mansuy, I. M., and Dutar, P. (2003) Different phosphatase-dependent mechanisms mediate long-term depression and depotentiation of long-term potentiation in mouse hippocampal CA1 area. Eur. J. Neurosci. $18,1279-1285$.

Kamprath, K., Marsicano, G., Tang, J., Monory, K., Bisogno, T., Marzo, V. D., Lutz, B., and Wotjak, C. T. (2006). Cannabinoid CB1 receptor mediates fear extinction via habituation-like processes. J. Neurosci. 26, 6677-6686.

Krasne, F. B., and Teshiba, T. M. (1995) Habituation of an invertebrate escape reflex due to modulation by higher centers rather than local events. Proc. Natl. Acad. Sci. U.S.A. 92, 3362-3366.

Li, H. L., Huang, B. S., Vishwasrao, H. Sutedja, N., Chen, W., Jin, I., Hawkins, R. D., Bailey, C. H., and Kandel, E. R.
(2009). Dscam mediates remodeling of glutamate receptors in Aplysia during de novo and learning-related synapse formation. Neuron 61, 527-540.

Li, Q., Roberts, A. C., and Glanzman, D. L. (2005). Synaptic facilitation and behavioral dishabituation in Aplysia: dependence upon release of $\mathrm{Ca}^{2+}$ from postsynaptic intracellular stores, postsynaptic exocytosis and modulation of postsynaptic AMPA receptor efficacy. J. Neurosci. 25, 5623-5637.

Lin, C.-H., Yeh, S.-H., Leu, T.-H., Chang, W.-C., Wang, S.-T., and Gean, P.-W. (2003). Identification of calcineurin as a key signal in the extinction of fear memory. J. Neurosci. 23, 1574-1579.

Lin, X. Y., and Glanzman, D. L. (1996). Long-term depression of Aplysia sensorimotor synapses in cell culture: inductive role of a rise in postsynaptic calcium. J. Neurophysiol. 76, 2111-2114.

Malenka, R. C., and Bear, M. F. (2004). LTP and LTD: an embarrassment of riches. Neuron 44, 5-21.

McSweeney, F. K., and Swindell, S. (2002). Common processes may contribute to extinction and habituation. J. Gen. Psychol. 129, 364.

Montarolo, P. G., Kandel, E. R., and Schacher, S. (1988). Long-term heterosynaptic inhibition in Aplysia. Nature 333, 171-174.

Mulkey, R. M., Endo, S., Shenolikar, S., and Malenka, R. C. (1994). Involvement of a calcineurin/inhibitor-1 phosphatase cascade in hippocampal long-term depression. Nature 369, 486-488.

Rankin, C. H., Abrams, T., Barry, R. J., Bhatnagar, S., Clayton, D. F., Colombo, J., Coppola, G., Geyer, M. A., Glanzman, D. L., Marsland, S., McSweeney, F. K., Wilson, D. A., Wu, C. F., and Thompson, R. F. (2009). Habituation revisited: an updated and revised description of the behavioral characteristics of habituation. Neurobiol. Learn. Mem. 92, 135-138. 
Schafe, G. E. (2008). Rethinking the role of L-type voltage-gated calcium channels in fear memory extinction. Learn. Mem. 15, 324-325.

Schreiber, S. L., and Crabtree, G. R. (1992). The mechanism of action of cyclosporin A and FK506. Immunol. Today 13, 136-142.

Schwartz, J.H., Castellucci, V.F., and Kandel, E. R. (1971). Functioning of identified neurons and synapses in abdominal ganglion of Aplysiain absence of protein synthesis. J. Neurophysiol. 34, 939-953.

Sehgal, P. B., and Tamm, I. (1978). Halogenated benzimidazole ribosides : novel inhibitors of RNA synthesis. Biochem. Pharmacol. 27, 2475-2485.

Sierra-Paredes, G., and Sierra-Marcuño, G. (2008). Ascomycin and FK506: pharmacology and therapeutic potential as anticonvulsants and neuroprotectants. CNS Neurosci. Ther. 14, 36-46.

Sutton, M. A., and Carew, T. J. (2002). Behavioral, cellular, and molecular analysis of memory in Aplysia I: intermediate-term memory. Integr. Comp. Biol. 42, 725-735.

Suzuki, A., Josselyn, S. A., Frankland, P.W., Masushige, S., Silva, A. J., and Kida, S. (2004). Memory reconsolidation and extinction have distinct temporal and biochemical signatures. J. Neurosci. 24, 4787-4795.

Suzuki, A., Mukawa, T., Tsukagoshi, A., Frankland, P. W., and Kida, S. (2008). Activation of LVGCCs and CB1 receptors required for destabilization of reactivated contextual fear memories. Learn. Mem. 15, 426-433.

Thompson, R. F., and Spencer, W. A. (1966). Habituation: a model phenomenon for the study of neuronal substrates of behavior. Psychol. Rev. 73, 16-43.

Yamaguchi, Y., Wada, T., and Handa, H. (1998). Interplay between positive and negative elongation factors: drawing a new view of DRB. Genes Cells 3, 9-15.

Conflict of Interest Statement: The authors declare that the research was conducted in the absence of any commercial or financial relationships that could be construed as a potential conflict of interest.
Received: 09 July 2010; paper pending published: 01 September 2010; accepted: 12 November 2010; published online: 29 November 2010.

Citation: Esdin J, Pearce K and Glanzman DL (2010) Long-term habituation of the gill-withdrawal reflex in Aplysia requires gene transcription, calcineurin and L-type voltage-gated calcium channels. Front. Behav. Neurosci. 4:181. doi: 10.3389/ fnbeh.2010.00181

Copyright (C) 2010 Esdin, Pearce and Glanzman. This is an open-access article subject to an exclusive license agreement between the authors and the Frontiers Research Foundation, which permits unrestricted use, distribution, and reproduction in any medium, provided the original authors and source are credited. 